\title{
Exciton dephasing in lead sulfide quantum dots by $X$-point phonons
}

\author{
Francesco Masia, ${ }^{1,2}$ Wolfgang Langbein, ${ }^{1, *}$ Iwan Moreels, ${ }^{3}$ Zeger Hens, ${ }^{3}$ and Paola Borri ${ }^{2, \dagger}$ \\ ${ }^{1}$ School of Physics and Astronomy, Cardiff University, The Parade, Cardiff CF24 3AA, United Kingdom \\ ${ }^{2}$ School of Biosciences, Cardiff University, Museum Avenue, Cardiff CF10 3AX, United Kingdom \\ ${ }^{3}$ Department of Inorganic and Physical Chemistry, Ghent University, Krijgslaan 281-S3, B-9000 Ghent, Belgium
}

(Received 11 May 2011; published 31 May 2011)

\begin{abstract}
Using a sensitive transient four-wave mixing technique in heterodyne detection, we measured the ground-state excitonic dephasing in $\mathrm{PbS}$ colloidal quantum dots of 4-6-nm diameter in the temperature range from 5 to $100 \mathrm{~K}$. We observe an ultrafast ( $<100 \mathrm{fs}$ ) initial decay followed by a component of about $300 \mathrm{fs}$ at $5 \mathrm{~K}$, which becomes faster with decreasing dot size and increasing temperature. This dynamics is attributed to pure dephasing of phonon-assisted transitions in the peculiar band structure of $\mathrm{PbS}$, which allows coupling to phonons with wave vectors both near the Brillouin zone center and near the (100) zone edge, the $X$ point. We estimate an upper limit of $7 \%$ for the weight of the zero-phonon line with 2.6-ps spin-flip limited dephasing.
\end{abstract}

DOI: 10.1103/PhysRevB.83.201309

PACS number(s): 78.67.Hc, 42.50.Md, 78.47.nj

The dephasing time inversely proportional to the homogeneous linewidth of an optical transition is a fundamental physical quantity in light-matter interaction. It was recognized about a decade ago that semiconductor quantum dots (QDs), in which carriers are confined in all three directions, exhibit a peculiar coupling of excitons with phonons as a result of the local lattice distortion associated with the excitonic excitation., ${ }^{1,2}$ This coupling gives rise to an excitonic homogeneous line shape at low temperatures consisting of a Lorentzian zero-phonon line (ZPL) superimposed onto a broad band of phonon-assisted transitions and, in turn, a strongly nonexponential dephasing. ${ }^{2}$

Among the various types of semiconductor quantum dots, $\mathrm{Pb} X(X=\mathrm{S}, \mathrm{Se})$ colloidal QDs (CQDs) have attracted much interest in recent literature due to their emission in the near infrared and their peculiar band structure. The effective masses of electrons and holes in these system are similar and relatively small, resulting in an exciton Bohr radius (46 $\mathrm{nm}$ for PbSe and $18 \mathrm{~nm}$ for $\mathrm{PbS}$ ) that is large compared to the typical QD sizes. Hence $\mathrm{PbX}$ dots are considered a model system for QDs with strong confinement. Moreover, both the maximum of the valence band and the minimum of the conduction band are at the $L$ point of the Brillouin zone, resulting in a 64-fold-degenerate ground-state exciton compared to the eightfold degeneracy in spherical QDs with a direct band gap at the $\Gamma$ point (e.g., InAs, InP, CdS, CdSe). ${ }^{3}$ Furthermore the exciton radiative lifetime is in the microsecond range, ${ }^{3}$ considerably longer than in other QDs, opening the prospect of ultralong radiative-lifetime limited dephasing times.

While the homogeneous line shape of excitons in III-V and II-VI QDs, epitaxially grown and colloidal, has been measured and calculated extensively in the literature, ${ }^{1,2,4,5}$ to date there have been only few works on $\mathrm{Pb} X \mathrm{CQDs}$. Low-temperature ensemble photoluminescence (PL) gave an upper limit of $30 \mathrm{meV}$ for the homogeneous linewidth in PbSe CQDs. ${ }^{6}$ Single-dot PL spectroscopy, ${ }^{1}$ commonly used to determine the homogeneous linewidth, is limited by the small radiative emission rate and the high detector noise in the near-infrared wavelength region of $\mathrm{PbX}$ emission. ${ }^{7}$ Moreover, spectral diffusion, a variation of the QD transition frequency over time from slow fluctuations of the QD environment, severely limits the ability to measure the intrinsic homogeneous linewidth in single-dot PL experiments. Recently, the PL linewidth of single $\mathrm{PbS}$ CQDs at room temperature emitting around $800 \mathrm{~nm}$ was found to be $\sim 100 \mathrm{meV}$, increasing with decreasing emission wavelength. ${ }^{7}$ Scanning tunneling spectroscopy (STS) at $5 \mathrm{~K}$ on single 4.3-nm PbSe CQDs with $\sim 1 \mathrm{eV}$ band gap showed $\sim 25$-meV full width at half maximum (FWHM) of the electron and hole resonances. ${ }^{8}$ These linewidths are larger than in $\mathrm{CdSe}$ CQDs of similar size, suggesting a faster dephasing; however, they could be affected by spectral diffusion and intervalley level splitting. The low-temperature homogeneous line shape and exciton dephasing time in $\mathrm{Pb} X$ CQDs is thus unknown.

In this work, we have measured the ground-state exciton dephasing in PbS CQDs in the temperature range from 5 to $100 \mathrm{~K}$ as a function of dot size using a sensitive transient four-wave mixing (FWM) technique in heterodyne detection, which overcomes the limitations of spectral diffusion and inhomogeneous broadening. We investigated five ensembles of core-only PbS CQDs, with fundamental excitonic absorption peaks at wavelengths between 1.09 and $1.49 \mu \mathrm{m}$ and corresponding average dot diameters from 3.7 to $5.7 \mathrm{~nm}$ (see Fig. 1). Each ensemble was dispersed in a polystyrene film and held in a cold-finger cryostat between quartz plates for temperature-dependent measurements. The PbS CQDs were synthesized based on the procedure of Ref. 9 .

In the FWM experiment, we use three pulse trains of 150-fs duration at 76- $\mathrm{MHz}$ repetition rate with a wavelength resonant to the fundamental exciton absorption of the CQDs (see Fig. 1). To increase the detection sensitivity we implemented both a heterodyne $\mathrm{e}^{2,10}$ and a directional selection geometry (see inset in Fig. 2), resulting in a large dynamic range of 8 orders of magnitude in FWM intensity. The first pulse $\left(P_{1}\right)$ induces a coherent polarization in the sample, which after a delay $\tau_{12}$ is converted into a density grating by the second pulse $\left(P_{2}\right)$. The third pulse $\left(P_{3}\right)$, delayed by $\tau_{23}$ from $P_{2}$, is diffracted and frequency shifted by this moving density grating, providing the FWM field that is detected by its interference with a reference pulse of adjustable delay. Pulses were linearly polarized horizontally $(\mathrm{H})$ or vertically $(\mathrm{V})$. In an inhomogeneously broadened ensemble the FWM signal is a photon echo ${ }^{11}$ in real time (delayed by $\tau_{12}$ from the third pulse). Spectral diffusion does not affect the photon echo since it is negligible during the 


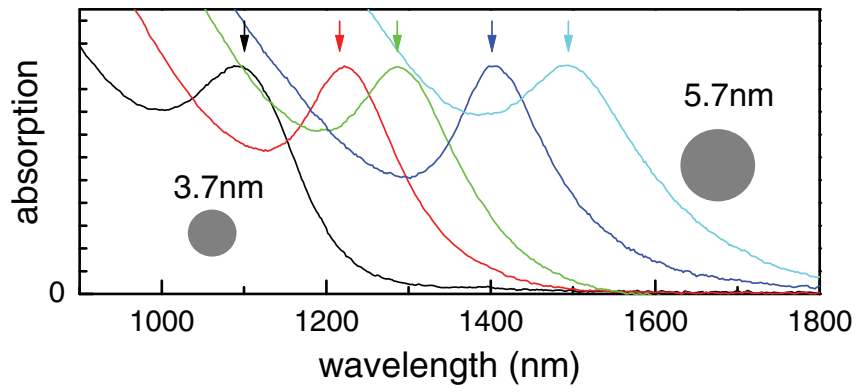

FIG. 1. (Color online) Normalized linear absorption coefficient of the investigated $\mathrm{PbSCQDs}$ measured in $\mathrm{C}_{2} \mathrm{Cl}_{4}$. Average dot diameters are (from left to right) 3.7, 4.3, 4.6, 5.2, and $5.7 \mathrm{~nm}$. The excitation laser energies used in the transient FWM experiment on these samples are marked by arrows.

time scale $\tau_{12}$. The microscopic dephasing is inferred from the decay of the time-integrated photon echo versus $\tau_{12}$.

In Fig. 2 the time-integrated FWM (TIFWM) field amplitude for the 4.6-nm CQDs is shown as a function of $\tau_{12}$ for different temperatures. Measurements are taken for $\tau_{23}=2 \mathrm{ps}$ to avoid nonresonant nonlinear effects at time overlap and in the HHVV polarization configuration of $P_{1}, P_{2}, P_{3}$, and reference to suppress background from detector nonlinearities. ${ }^{10}$ A change in $\tau_{23}$ only changes the total FWM amplitude due to the decay of the density grating but does not modify the dynamics versus $\tau_{12}$ (see dotted line in Fig. 2 for $\tau_{23}=100 \mathrm{ps}$ ). Remarkably, even at $5 \mathrm{~K}$ we observe a dominant ultrafast initial decay within the $\sim 200$-fs autocorrelation of the exciting pulses, followed by a resolved component with an exponential decay time of a few hundred femtoseconds. Such decay is more than 6 orders of magnitude shorter than the $\sim 1-\mu$ s exciton lifetime observed in the time-resolved PL of this sample. To quantify the dephasing, we have fitted the resolved component with a hyperbolic secant function $A_{1} / \cosh \left(2 \tau_{12} / t_{1}\right)$ multiplied

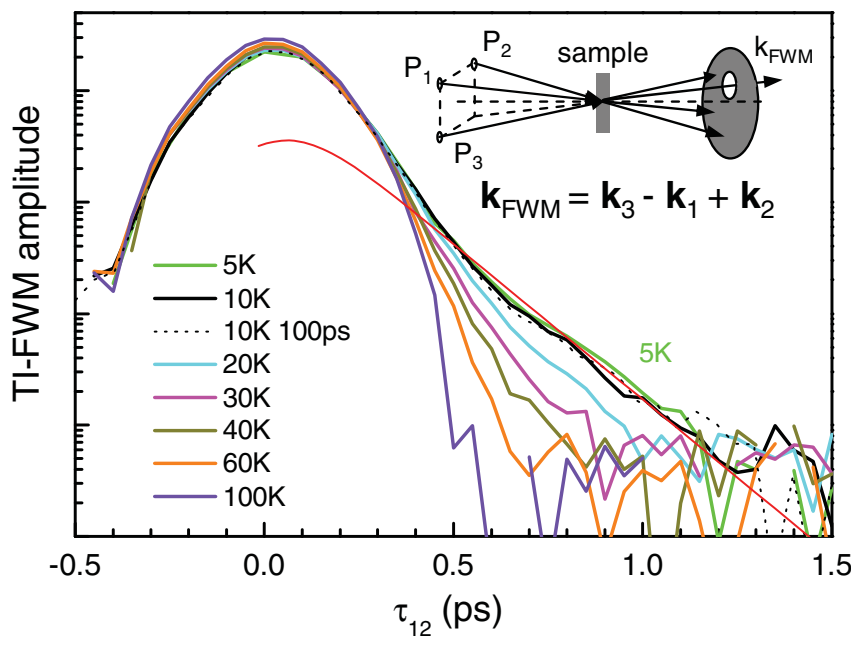

FIG. 2. (Color online) Time-integrated FWM field amplitude versus delay $\tau_{12}$ for the 4.6-nm CQDs at different temperatures and at $\tau_{23}=2$ ps delay between the second and third pulses. The dynamics for $\tau_{23}=100 \mathrm{ps}$ at $10 \mathrm{~K}$ is shown as a dotted line. The thin solid line is a fit to the data at $5 \mathrm{~K}$ using a hyperbolic secant function. In the inset the directional geometry used for the excitation and detection of the FWM field is sketched.
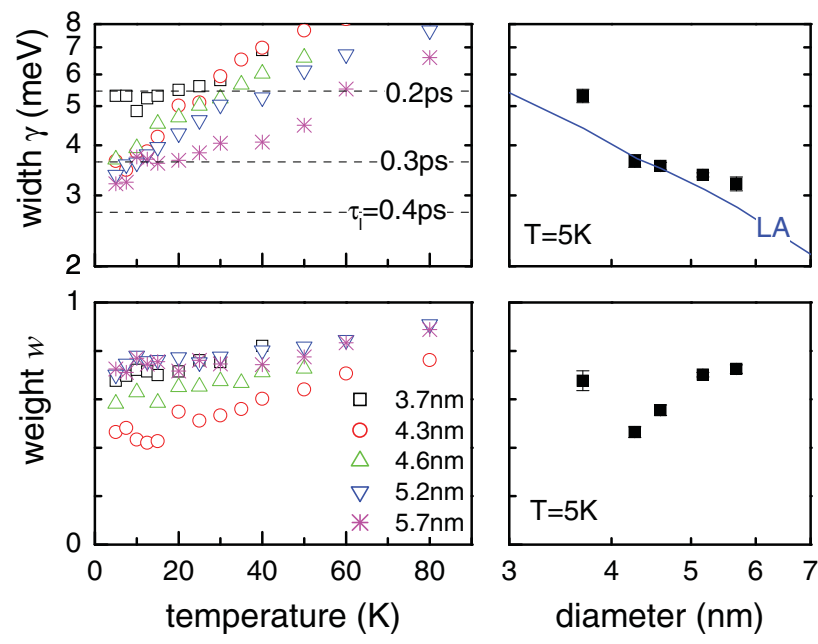

FIG. 3. (Color online) (left) Temperature dependence of the longer component for different CQD sizes, characterized by the homogeneous linewidth $\gamma$ (top) and the weight $w$ (bottom). (right) The same as in the left column, but with dependence on the CQD size at low temperature $T=5 \mathrm{~K}$. The solid line shows the estimated FWHM of the LA phonon band (see text).

by a step function to include causality. This function has an exponential tail that fits the experimental data (see Fig. 2), and its analytical Fourier transform (also a hyperbolic secant) can be used to represent the homogeneous line shape with FWHM $\gamma=1.67 \hbar / t_{1}$. If the exponential decay is attributed to a Lorentzian line shape, its FWHM is $2 \hbar / t_{1}$. However, as we will argue later, the physical origin of this component is likely to be phonon-assisted transitions, which are not well described by a Lorentzian. To quantify $A_{1}$ we have fitted the difference between the measured FWM dynamics and the subresolution autocorrelation-like contribution, the latter taken proportional to the FWM dynamics measured at $100 \mathrm{~K}$. The proportionality factor was estimated from the best fit to the data at $5 \mathrm{~K}$ and kept constant versus temperature.

The temperature dependence of the resolved component is similar for all CQDs investigated, as shown in Fig. 3, where we plot its width $\gamma$ and its weight, defined as ${ }^{12} w=\sqrt[3]{A_{1} / A_{0}}$, where $A_{0}$ is the FWM amplitude measured at $\tau_{12}=0$. The width is in the several meV range at low temperatures and increases by a factor of $2-3$ up to $80 \mathrm{~K}$. For comparison, in InGaAs self-assembled QDs ${ }^{2,12}$ and CdSe/ZnS CQDs ${ }^{13}$ the $\mathrm{ZPL}$ width is in the $1-10-\mu \mathrm{eV}$ range at $5 \mathrm{~K}$ and rapidly increases by nearly 3 orders of magnitudes to the meV range at $100 \mathrm{~K}$ while significantly decreasing its weight.

Extrinsic mechanisms, such as charging or surface and trap states, are unlikely to influence the measured dephasing since the long PL lifetime indicates that trapping of carriers is not of importance, which is confirmed by similar lifetimes of core and core-shell PbS QDs. ${ }^{10}$ Furthermore, multicarrier effects, such as charged excitons or multiexcitons, give rise to a population decay on the $10-100$-ps timescale due to Auger recombination, ${ }^{14}$ which is not observed in the density dynamics. We found that such processes are important for time-averaged excitation intensities $>1 \mathrm{KW} / \mathrm{cm}^{2}$ (Ref. 10), 1 order of magnitude larger than used in the present experiment. 


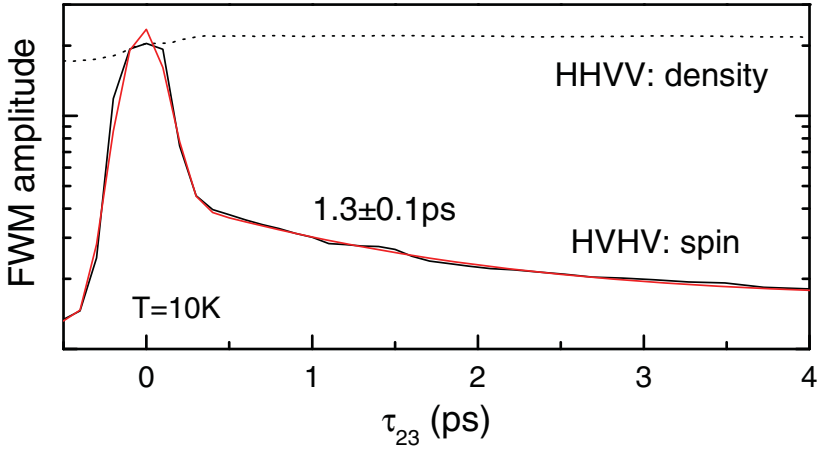

FIG. 4. (Color online) FWM dynamics of the 4.6-nm CQDs versus $\tau_{23}$ for $\tau_{12}=0$ and different polarization configurations. A fit to the CPTG dynamics (thin line) and the corresponding spin-relaxation time are also shown.

A possible mechanism responsible for a fast dephasing of the ZPL in PbS CQDs is the phonon-assisted thermalization between the different fine-structure states of the excitonic ground state, which are split by intervalley scattering and exchange interaction and have different angular momentum projections. ${ }^{3}$ Such spin relaxation can be measured using cross-polarized transient-grating (CPTG) experiments, ${ }^{15}$ which we performed by cross polarizing $P_{1}$ and $P_{2}$ and measuring the FWM amplitude versus $\tau_{23}$ at $\tau_{12}=0$. As shown in Fig. 4 we find that in the polarization configuration HVHV the CPTG dynamics shows an instantaneous contribution at zero delay, influenced by the density grating generated by $P_{3}$ and probed by $P_{2}$, followed by a component with a $\sim 1.3$-ps decay time and a long-lived component. In comparison, the density dynamics probed in HHVV configuration exhibits a much slower dynamics given by the exciton population relaxation. The measured $\sim 1.3$-ps exciton spin-relaxation time at $10 \mathrm{~K}$ is consistent with the results of Ref. 15 for similar dot sizes. The corresponding dephasing time of $\sim 2.6 \mathrm{ps}$, twice the spin relaxation time, corresponds to a linewidth of $\sim 0.5 \mathrm{meV}$, an order of magnitude below the measured values (see Fig. 3). We therefore conclude that spin relaxation is not dominating the observed dephasing. Since the low-temperature ZPL dephasing is given by spin relaxation and the much longer radiative decay, the resolved dephasing component cannot be due to the ZPL. This implies that the ZPL contribution to the FWM is below our detection sensitivity, allowing us to deduce an upper limit of the ZPL weight in the line shape. Considering the dynamic range of 4 orders of magnitude in FWM field amplitude in our experiment and the cubic relationship between ZPL weight and FWM amplitude ${ }^{12}$ and assuming a spin-flip limited ZPL dephasing time of $2.6 \mathrm{ps}$, we find an upper limit of $7 \%$ at $10 \mathrm{~K}$.

The resolved dephasing component should consequently be attributed to phonon-assisted optical transitions. The effect of such transitions on the FWM dynamics from QDs was observed in self-assembled InGaAs/GaAs QDs at low temperature ${ }^{2,12}$ as an initial decay on the picosecond timescale corresponding to a non-Lorentzian band of a few $\mathrm{meV}$ width in the homogeneous line shape. Its physical origin is the modification of the adiabatic potential for the nuclear motion due to the localized excitonic excitation, enabling optical transitions with absorption and emission of phonons, similar to rotovibrational bands in molecules. Such an acoustic-phonon band was also reported in CdSe/ZnS CQDs. ${ }^{5,13}$ Since the exciton-phonon interaction does not lead to a change in the excitonic population, it accounts for pure dephasing and is described theoretically by the independent Boson model. ${ }^{1,16}$ The width of this acoustic phonon band is inversely proportional to the size of the excitonic wave function since it is determined by the wave-vector range of the excitonic wave function, ${ }^{16}$ or, more intuitively, by the time the excited phonons take to leave the QD. We indeed observe (see Fig. 3, top right) that $\gamma$ is increasing with decreasing nanoparticle radius.

To quantitatively estimate the expected dynamics, we calculated the electron-phonon coupling form factor $f_{q}$ [Eq. (34) in Ref. 17] using as electron and hole states the ground state of a cubic infinite potential well of side length equal to the dot diameter. The resulting coupled regions within the bulk $\mathrm{PbS}$ phonon dispersion ${ }^{18}$ are highlighted in Fig. 5. The energy range of LA phonons (solid line in Fig. 3) shows good agreement with the measured $\gamma$. However, when evaluating the resulting FWM dynamics for deformation potential coupling of LA phonons using Eqs. (A1) and (A2) in Ref. 17, we found a ZPL weight of about 0.85 at $10 \mathrm{~K}$, which is much larger than the estimated upper limit of 0.07 , showing that other phonon modes contribute significantly.

In CdSe CQDs of similar core size, besides the acoustic phonon band, LO phonon-assisted transitions are observed in the PL spectrum with an energy separation around $26 \mathrm{meV}$ and a few meV linewidth. ${ }^{13}$ The CdSe band structure has a direct gap at the zone-center $\Gamma$, resulting in electron and hole wave functions localized near $\Gamma$. This implies a wave-vector
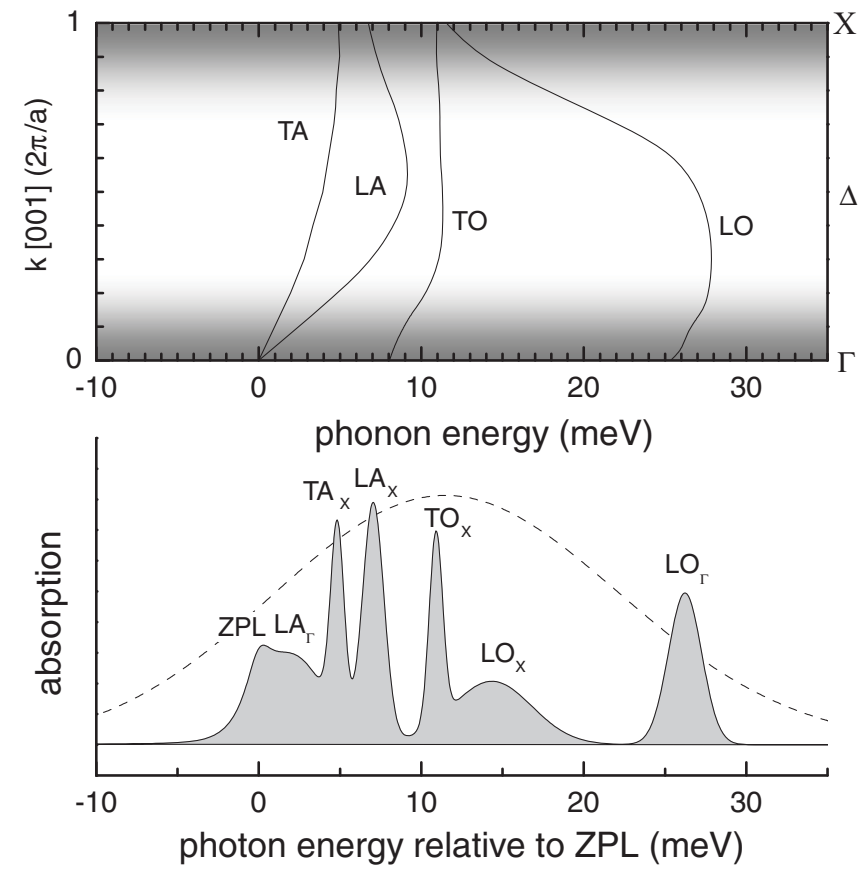

FIG. 5. (top) Phonon dispersion in $\mathrm{PbS}^{18}$ from $\Gamma$ to $X$ along $\Delta$. The ranges coupled to the excitonic transition of a 4.6-nm PbS CQD are indicated in gray. (bottom) Sketch of the resulting absorption profile. The relative weights of the phonon branches are chosen equally, neglecting transversal phonons at the $\Gamma$ point. The excitation pulse spectrum is shown as a dashed line. 
selection of the coupled phonons near $\Gamma$, at which both optical and acoustic phonons have rather well-defined energies of $26 \mathrm{meV}$ and a few meV, respectively. In PbS CQDs instead, electron and hole wave functions contain contributions of the four $L$ valleys. Accordingly, phonon-assisted transitions involve also phonons near the difference between any two $L$ points at $\mathbf{k}=\frac{2 \pi}{a}(100)$ ( $X$ points) with the lattice constant $a=0.5936 \mathrm{~nm}$ of the $\mathrm{PbS}$ face-centered-cubic lattice. The absorption line shape of a single exciton level of an individual QD thus consists of a series of phonon-assisted bands, as sketched in the bottom panel of Fig. 5. Although a quantitative theory of the coupling with these various phonon modes is presently not available, the large phonon density of states close to the $X$ point is likely to result in strong phonon-assisted transitions. The ZPL is included as a Lorentzian peak broadened by the spinflip relaxation with a weight of $7 \%$. Hence, phonon-assisted transitions are distributed over an energy range of about $30 \mathrm{meV}$, and this picture is enriched by multiphonon processes, creating an even larger spectral range. The mutual dephasing of the individual phonon bands that are excited within the spectral width of the pulse (dashed line in Fig. 5) accounts for the observed subresolution component in the FWM.
In conclusion, we measured a two-component subpicosecond dephasing in $\mathrm{PbS}$ colloidal quantum dots at $5 \mathrm{~K}$, more than 6 orders of magnitude faster than the microsecond excitonic lifetime in these systems. This measurement of the intrinsic dephasing time in chalcogenide quantum dots at low temperature is key to their application in optoelectronics devices from lasers and solar cells to quantum information processing. The proposed interpretation by phonon-assisted bands involving phonons at the $X$ point should stimulate future experiments, such as low-temperature single-dot spectroscopy or transient phonon echo with $<30$-fs resolution and the development of a quantitative theory extending presently known models.

F.M. acknowledges financial support from the European Union (Marie Curie Grant Agreement No. PIEF-GA-2008220901) and the Welcome Trust (VIP award). P.B. acknowledges the EPSRC UK Research Council (Grant No. $\mathrm{EP} / \mathrm{I005072/1).} \mathrm{I.M.} \mathrm{is} \mathrm{a} \mathrm{researcher} \mathrm{with} \mathrm{the} \mathrm{FWO-Vlaanderen.}$ Z.H. acknowledges BelSPo (IAP 6.10, photonics@be) for funding this research. *langbeinww@ cardiff.ac.uk

†borrip@cardiff.ac.uk

${ }^{1}$ L. Besombes, K. Kheng, L. Marsal, and H. Mariette, Phys. Rev. B 63, 155307 (2001).

${ }^{2}$ P. Borri, W. Langbein, S. Schneider, U. Woggon, R. L. Sellin, D. Ouyang, and D. Bimberg, Phys. Rev. Lett. 87, 157401 (2001).

${ }^{3}$ J. M. An, A. Franceschetti, and A. Zunger, Nano Lett. 7, 2129 (2007).

${ }^{4}$ E. A. Muljarov and R. Zimmermann, Phys. Rev. Lett. 93, 237401 (2004).

${ }^{5}$ P. Palinginis, H. Wang, S. V. Goupalov, D. S. Citrin, M. Dobrowolska, and J. K. Furdyna, Phys. Rev. B 70, 73302 (2004).

${ }^{6}$ J. M. Harbold and F. W. Wise, Phys. Rev. B 76, 125304 (2007).

${ }^{7}$ J. J. Peterson and T. D. Krauss, Nano Lett. 6, 510 (2006).

${ }^{8}$ P. Liljeroth, P. A. Zeijlmans van Emmichoven, S. G. Hickey, H. Weller, B. Grandidier, G. Allan, and D. Vanmaekelbergh, Phys. Rev. Lett. 95, 086801 (2005).
${ }^{9}$ I. Moreels, K. Lambert, D. Smeets, D. De Muynck, T. Nollet, J. C. Martins, F. Vanhaecke, A. Vantomme, C. Delerue, G. Allan, and Z. Hens, ACS Nano 3, 3023 (2009).

${ }^{10}$ F. Masia, I. Moreels, Z. Hens, W. Langbein, and P. Borri, Phys. Rev. B 82, 155302 (2010).

${ }^{11}$ S. Mukamel, Principles of Nonlinear Optical Spectroscopy (Oxford University Press, New York, 1999).

${ }^{12}$ P. Borri, W. Langbein, U. Woggon, V. Stavarache, D. Reuter, and A. D. Wieck, Phys. Rev. B 71, 115328 (2005).

${ }^{13}$ M. J. Fernée, B. N. Littleton, S. Cooper, H. Rubinsztein-Dunlop, D. E. Gómez, and P. Mulvaney, J. Phys. Chem. C 112, 1878 (2008).

${ }^{14}$ I. Robel, R. Gresback, U. Kortshagen, R. D. Schaller, and V. I. Klimov, Phys. Rev. Lett. 102, 177404 (2009).

${ }^{15}$ J. C. Johnson, K. A. Gerth, Q. Song, J. E. Murphy, A. J. Nozik, and G. D. Scholes, Nano Lett. 8, 1374 (2008).

${ }^{16}$ A. Vagov, V. M. Axt, T. Kuhn, W. Langbein, P. Borri, and U. Woggon, Phys. Rev. B 70, 201305(R) (2004).

${ }^{17}$ A. Vagov, V. M. Axt, and T. Kuhn, Phys. Rev. B 67, 115338 (2003).

${ }^{18}$ M. M. Elcombe, Proc. R. Soc. London, Ser. A 300, 210 (1967). 